\title{
Diminishing activity of recent solar cycles (22-24) and their impact on geospace
}

\author{
Bharati Kakad*, Amar Kakad, Durbha Sai Ramesh, and Gurbax S. Lakhina \\ Indian Institute of Geomagnetism, New Panvel, Navi Mumbai, 410218, India
}

Received 20 March 2018 / Accepted 26 November 2018

\begin{abstract}
This study examines the variation of different energies linked with the Sun and the Earth's magnetosphere-ionosphere systems for solar cycles (SCs) 22-24 for which the gradual decrease in the solar activity is noticed. Firstly, we investigated the variation of solar magnetic energy density (SMED) for SCs 21-24 and its relation to the solar activity. We observed distinct double peak structures in SMED for the past four SCs, 21-24. This feature is consistent with noticeable asymmetry in their two peaks. For SCs 22-24 a significant decrease is observed in the integrated SMED of each SC. This reduction is $37 \%$ from SCs 22 to 23 and 51\% from SCs 23 to 24, which indicates substantial weakening of Sun's magnetic field for SC 24. Also, the magnetic, kinetic, and thermal energy densities at the Earth's bow-shock nose are found to be considerably low for the SC 24. We examined the solar wind Alfven speed, magnetosonic Mach number, solar wind-magnetosphere energy coupling parameter $(\varepsilon)$, and the Chapman-Ferraro magnetopause distance $\left(L_{\mathrm{CF}}\right)$ for the SCs $22-24$. The estimated maximum stand-off magnetopause distance is larger for SC $24\left(L_{\mathrm{CF}} \leq 10.6 R_{\mathrm{E}}\right)$ as compared to SC $23\left(L_{\mathrm{CF}} \leq 10.2 R_{\mathrm{E}}\right)$ and SC $22\left(L_{\mathrm{CF}} \leq 9.8 R_{\mathrm{E}}\right)$. The solar wind Alfven speeds during SCs 22 and 23 are in the same range and do not exceed $\approx 73 \mathrm{~km} / \mathrm{s}$ whereas, it is below $57 \mathrm{~km} / \mathrm{s}$ for SC 24 . A lower bound of solar wind magnetosonic Mach number for SC 24 is larger $(M \geq 6.9)$ as compared to SC $22(M \geq 5.9)$ and SC $23(M \geq 6)$. We noticed weakening in the energy coupling parameter for SC 24 , which resulted in substantial $(15 \%-38 \%)$ decrease in average strength of high latitude ionospheric $(A E)$, low latitude magnetospheric $(D s t)$ and equatorial ionospheric $(E E J)$ current systems in comparison with SC 23 . Subsequently, a reduction of $\approx 30 \%$ is manifested in the high latitude Joule heating for SC 24. Overall this study indicates the significant step down in various energies at Sun, Earth's bow-shock, and near Earth environment for current SC 24, which will have important implication on our Earth's atmosphere-ionosphere-magnetosphere system.
\end{abstract}

Keywords: solar cycle / magnetic fields / photosphere / magnetosphere / solar wind

\section{Introduction}

The Sun is the main source of energy for our Earth, and its variability influences the Earth's atmosphere, ionosphere, magnetosphere systems, and climate, which is evident from several studies (McComas et al., 2008; Ermolli et al., 2013; Dudok de Wit \& Bruinsma, 2017). It is known that geomagnetic storm, the most important element of the space weather, is directly related to the activity of the Sun (Tsurutani et al., 2003; Le et al., 2012; Hajra et al., 2016). Thus, the scientific community keeps a constant watch on the Sun's activity to know more about the nature of present and future solar activity. In recent past, the predictions of peak activity of SC 23 proposed by

\footnotetext{
*Corresponding author: bkakad9@gmail.com
}

different methods have failed (Li et al., 2001; Petrovay, 2010) and it was found to be smaller as compared to SC 22. Also, remarkable changes in the latitudinal distribution of highand low-speed solar wind flow were observed for SC 22 and 23 (Manoharan, 2012). Later, the end time of SC 23 was also sufficiently prolonged to give rise to a deep extended low solar minimum period between SC 23 and 24. This anomalous behaviour was attributed to the Sun's meridional plasma flow (Janardhan et al., 2010; Nandy et al., 2011). The considerable weakening of the solar polar magnetic field is noticed during this period (Schrijver \& Liu, 2008; Hathaway \& Upton, 2014). A recent study using information theory suggests that the polar solar magnetic fields from the last three or more SCs can affect the production of the peak smooth sunspot number (SSN) of the subsequent cycle (Wing et al., 2018), it means 
that present decrease in solar magnetic field may have longterm implications on the formation of SSN. The extended minimum of SC 23/24 gave an opportunity to explore the behaviour of various ambient parameters in the near Earth space during extremely low solar activity periods. Noticeable change in the Earth's atmosphere has been observed in the prolonged minimum of SC 24 (de Toma et al., 2010; Solomon et al., 2013). This is an alarming situation as it hints towards the level of changes that may be encountered in the Earth's ionospheremagnetosphere system during such extremely low solar activity periods.

The current SC 24 is witnessing the further weakening of the solar activity. It is reported that the occurrence of geomagnetic storms during ascending phase of SC 24 is lesser than SC 23 (Richardson, 2013), in spite of remarkable similarities in CME occurrences (Selvakumaran et al., 2016) during their ascending phases. Also, studies have shown that the solar photospheric magnetic fields and micro-turbulence levels in the heliosphere are decreasing since 1995 and it is still continuing (Janardhan et al., 2011; Bisoi et al., 2014; Janardhan et al., 2015a). A careful scrutiny of SC variations suggest that the characteristics of all SCs are unique. However, one noticeable feature is the gradual decrease in Sun's activity for the last three SCs. The peak smooth sunspot number (SSN) of 116.4 for the present SC 24 occurred during April 2014. This is small compared to the peak SSN of 232.9, 212.5 and 180.3 for SC 21, 22 and SC 23, respectively. These peak SSN are based on the newly revised Version-2 sunspot series (Clette et al., 2016). If we compare relative decrease in peak SSN of consecutive SCs starting from SC 21 (i.e., from SCs 21 to 22, 22 to 23, 23 to 24 ), then it is $8.7 \%, 15 \%$ and $35 \%$ for SCs 22,23 and 24 , respectively. So the present SC 24 exhibits the highest reduction in its activity as compared to SC 22 and SC 23. If we observe past $\mathrm{SCs}$, such gradual decrease in peak sunspot activity is encountered for SCs 8-10 (1833.8-1867.2) with peak SSN of 244.9, 219.9 , and 186.2, respectively, which corresponds to successive decrease of only $10 \%$ and $15 \%$ in the peak SSN from SC 8 to SC 10. Thus, it is evident that the solar activity has experienced significant decline for the last three solar cycles, 22-24, which is manifested in the solar magnetic field as well. The recent forecasting models suggest that the upcoming SC 25 would be still weaker (Gkana \& Zachilas, 2016; Kakad et al., 2017a).

A study by Yamauchi (2015) decreased reports the decrease in Sun-Earth coupling efficiency during recent past (i.e., 2005-2014) than those during the previous four decades (i.e., 1965-2004). Their study conclude that this observed decrease cannot be explained by existing magnetosphereionosphere coupling knowledge but rather it might be related to the unidentified mechanism connected to the long-term solar activity. In such a scenario, it is worth exploring the overall impact of the recent diminishing solar activity on the near Earth environment. It is also important to compare and quantify the variations in solar magnetic energy density (SMED; it is estimated from the mean solar magnetic field), solar wind parameters and corresponding energies, and their coupling with the Earth's ionosphere-magnetosphere system. There are not many attempts in this direction. One certainly expects to see the decrease in the various energies and the strength of different current systems in the vicinity of Earth due to a decrease in the solar activity. However, the expected decrease may not be linear. This raises the question of how do Earth's ionospheremagnetosphere systems configure themselves during periods of such extremely low solar activity and can we quantify these changes?

To address these questions, we estimated the energies for the recent SCs utilizing public domain data related to the Sun, magnetosphere, and ionosphere systems. In-addition, we used the magnetic field variations recorded at low-latitude magnetic observatories operated by our institute in Indian longitude. In order to understand possible linkages between SMED and solar wind interplanetary parameters and associated energies at the Earth's bow-shock nose, and its coupling to the magnetosphere-ionosphere system, we first focused on the variations in SMED and the attendant manifestations at the bow-shock nose carried through the solar wind plasma. Later, the overall energy coupling between the solar wind and Earth's magnetosphere and further energy transferred to the Earth's high latitude, and equatorial current systems are investigated. The present study provides the insights into the average ambient conditions prevailing on the Sun, inside and outside the Earth's magnetosphere for recent SCs. This information is crucial since the solar activity has progressively weakened over SCs 22-24 and such gradually declining solar activity may have short, and long time implications to the Earth's atmosphere-ionosphere-magnetosphere system. The details of the data sets used in this study with the methodologies adopted to arrive at various energies are described in Section 2. The results are presented in Section 3. The present study is summarized and concluded in Section 4.

\section{Data sets and energy estimates}

In the present study, various data sets available in the public domain have been used. The Version-2 monthly smoothed $\left(S_{\mathrm{ms}}\right)$ international sunspot number observations, which are available at http://www.sidc.be/silso/ are utilized to get information on solar activity. This is a newly revised sunspot series, which is the corrected version of the original Version-1 sunspot number series (Clette et al., 2016). Recently, Version-2 sunspot numbers are used by the researchers for SC studies (Zachilas \& Gkana, 2015). Monthly SSN for $j$ th month is given by the 13month weighted average centered on $j$ th month such that the half weights are assigned to ending months. This is a standard smoothing function, which is applied to the sunspot observations to remove the short time period variations. This monthly smoothed data of sunspot number is used to retrieve SC characteristics like ascent time $\left(T_{\mathrm{a}}\right)$, descent time $\left(T_{\mathrm{d}}\right)$, length $\left(T_{\text {cy }}\right)$, solar maximum $\left(S_{\max }\right)$, and solar minimum $\left(S_{\min }\right)$. We need to identify the start time $\left(t_{\mathrm{s}}\right)$, peak time $\left(t_{\mathrm{p}}\right)$, and end time $\left(t_{\mathrm{e}}\right)$ of a $\mathrm{SC}$ to estimate these characteristics. The mathematical minimum and maximum in the monthly SSN are identified and assigned to the start time and peak time of SC respectively (Kakad, 2011). If the same value of minimum (maximum) is encountered more than once in the monthly SSN then its first occurrence is treated as the time of minimum (maximum) for the corresponding SC. It should be noted that end time of nth SC is the start time for the $n+1$ th SC. The value of monthly SSN at the time of start and peak are treated as SC minimum 
B. Kakad et al.: J. Space Weather Space Clim. 2019, 9, A1

Table 1. SC characteristics namely start time, peak time, ascent time, descent time, length, peak sunspot number, and minimum sunspot number are given for SCs 21-24. Version-2 series of international sunspot number is used to obtain these characteristics.

\begin{tabular}{|c|c|c|c|c|c|c|c|}
\hline SC No. & Start time, $t_{\mathrm{s}}(\mathrm{yr})$ & Peak time, $t_{\mathrm{p}}(\mathrm{yr})$ & Ascent, time $T_{\mathrm{a}}(\mathrm{yr})$ & Descent time, $T_{\mathrm{d}}(\mathrm{yr})$ & Length, $T_{\mathrm{cy}}(\mathrm{yr})$ & $S_{\min }$ & $S_{\max }$ \\
\hline 22 & 1986.71 & 1989.87 & 3.17 & 6.50 & 9.67 & 13.50 & 212.50 \\
\hline 24 & 2008.96 & 2014.29 & 5.33 & - & - & 2.20 & 116.40 \\
\hline
\end{tabular}

$\left(S_{\min }\right)$, and maximum $\left(S_{\max }\right)$, respectively. These SC characteristics are compiled in Table 1 for SCs 21-24.

We have utilized a daily solar mean magnetic field for SCs 21-24, which is available at http://wso.stanford.edu. In addition, the SWP density $(N)$, velocity $(V)$, proton temperature $(T)$, interplanetary magnetic field $(B)$ at Earth's bow-shock nose of hourly sampling are used. This data is available at https:// omniweb.gsfc.nasa.gov. We have utilized the interplanetary solar wind information for SCs, 22-24. To understand the response of Earth's current systems, we looked into the proxies representing high latitude currents like the auroral electrojet $(A E)$, low latitude magnetospheric ring current (Dst), and equatorial ionospheric $E$-region current system known as equatorial electrojet $(E E J)$. The hourly observations of $A E$, and $D s t$ are obtained from http://wdc.kugi.kyoto-u.ac.jp. Whereas, to examined $E E J$ we used the hourly ground magnetic field data recorded at Trivandrum $\left(8.5^{\circ} \mathrm{N}, 77^{\circ} \mathrm{E}\right.$, dip latitude $\left.0.5^{\circ} \mathrm{N}\right)$, Tirunelveli $\left(8.7^{\circ} \mathrm{N}, 77.8^{\circ} \mathrm{E}\right.$, dip latitude $\left.0.6^{\circ} \mathrm{N}\right)$ and Alibaug $\left(18.6^{\circ} \mathrm{N}, 72.9^{\circ} \mathrm{E}\right.$, dip latitude $\left.14.1^{\circ} \mathrm{N}\right)$ for the period of 1985-2017. Both Tirunelveli and Trivandrum are located close to each other. Magnetic field data at Trivandrum is available from January 1985 to September 2000 and at Tirunelveli data is available from October 2000 to November 2017, respectively. These data sets are available on http://www.wdciig.res.in.

Here, we describe different parameters and the computation of various physical quantities carried out for the present study. As a first step, the daily solar magnetic field $\left(B_{\text {solar }}\right)$ is used to compute SMED employing the relation, $U_{\mathrm{SM}}=B_{\text {solar }}^{2} / 2 \mu_{0}$. The solar mean magnetic field represents the disk-averaged line-of-sight magnetic field that also reflects the polarity imbalance of the magnetic field on the Sun. It has contribution from both the large scale magnetic field structure (i.e., ambient magnetic field) and the active regions on Sun. In recent study Bose \& Nagaraju (2018) have shown that the variations in solar magnetic field has major $(89 \%)$ contribution from the ambient magnetic field and the contribution from other features is insignificant (11\%). It may be noted that SMED has undergone considerable gradual decrease from SC 22 to SC 24 (it is discussed in Sect. 3.1). So, in the present study we explored the variations of different physical quantities and energies related to Earth's magnetosphere-ionosphere system during this declining activity period of SCs 22-24. The Magnetic energy density gives information about the magnetic pressure. As we are using the average magnetic field of the Sun, it represents the average magnetic pressure at the Sun. The magnetic field of the Sun is controlled by the solar dynamo and hence it is an important physical parameter to track the solar activity. Later, using solar wind parameters at the Earth's bow-shock nose, we computed (i) magnetic energy density $\left(U_{\mathrm{m}}=B^{2} / 2 \mu_{0}\right)$, (ii) kinetic energy density or dynamic pressure $\left(U_{\mathrm{ke}}=m_{\mathrm{p}} N V^{2} / 2\right)$, (iii) thermal energy density $\left(U_{\text {th }}=N K_{\mathrm{B}}\left(T_{\mathrm{p}}+T_{\mathrm{e}}\right) / 2\right)$, (iv) Alfven speed,
$V_{\mathrm{A}}=B / \sqrt{N m_{\mathrm{p}} \mu_{0}}$, (v) ion thermal velocity, $V_{\mathrm{th}}=$ $\sqrt{K_{\mathrm{B}} T_{\mathrm{p}} / m_{\mathrm{p}}}$, (vi) magnetosonic Mach number, $M=V /$ $\sqrt{V_{\mathrm{A}}^{2}+V_{\mathrm{s}}^{2}}$, and (vii) SWP beta $(\beta)$, which is a ratio of thermal energy to magnetic energy $\left(\frac{U_{\text {th }}}{U_{\mathrm{m}}}\right)$ at five minute interval. Here, $V_{\mathrm{s}}=\sqrt{K_{\mathrm{B}}\left(T_{\mathrm{p}}+T_{\mathrm{e}}\right) / m_{\mathrm{p}}}$ is the acoustic speed. The standard symbols have their usual meanings, $K_{\mathrm{B}}$ is Boltzmann constant, $\varepsilon_{0}$ is permittivity of free space, $m_{\mathrm{p}}$ mass of proton. In earlier studies, it is reported that the electrons are slightly hotter than the ions in the SWP (Newbury et al., 1998). So one can assume $T_{\mathrm{p}} \sim T_{\mathrm{e}}$ in the computation of total thermal energy density of SWP. Being less massive, the electron's contribution to the kinetic energy can be neglected.

The magnetopause is the boundary at which solar wind kinetic pressure and the Earth's magnetic field pressure balance each other. The location of magnetopause can be estimated through Chapman-Ferraro magnetopause distance $L_{\mathrm{CF}}$ represented as (Sibeck et al., 1991),

$$
L_{\mathrm{CF}}=\left[\frac{2 B_{\mathrm{o}}^{2}}{\mu_{0} m_{\mathrm{p}} N V^{2}}\right]^{1 / 6} R_{\mathrm{E}} .
$$

Here, all quantities are in SI units, $R_{\mathrm{E}}$ is the Earth radius $(6371 \mathrm{~km}), B_{\mathrm{o}}$ is the magnetic field on surface of the Earth in equatorial plane $(30,000 \mathrm{nT})$. We estimated $L_{\mathrm{CF}}$ from the solar wind information at the bow-shock nose using above equation (1). Apart from this, the solar wind-magnetosphere energy coupling parameter used to understand the energy transfer to the magnetosphere. It should be noted that there are other coupling functions as well, which are summarized by Newell et al. (2007). In the present study, we have used the energy coupling parameter $\varepsilon$, which is proposed by Perreault \& Akasofu (1978), and it is given by,

$$
\varepsilon=\left[V B^{2} L_{\mathrm{CF}}^{2} \sin ^{4}\left(\frac{\theta}{2}\right)\right] \times 10^{7} .
$$

All quantities in equation (2) are in SI unit. Perreault \& Akasofu (1978) considered a value of $L_{\mathrm{CF}}=7 R_{\mathrm{E}}$, by assuming constant stand-off position of the magnetopause. But, it is known that the location of magnetopause can change depending on the solar wind pressure. So, in the above equation, the values of $L_{\mathrm{CF}}$ are taken from equation (1). This energy coupling function is used in many studies to understand the energy transfer to the Earth's magnetosphere (Koskinen \& Tanskanen, 2002; Guo et al., 2011). Here, $\varepsilon$ is expressed in Watt, and $\theta$ is a clock angle given by,

$$
\theta=\tan ^{-1}\left[\frac{\left|B_{y}\right|}{\left|B_{z}\right|}\right] \quad \text { for } B_{z}<0,
$$




$$
\theta=180+\tan ^{-1}\left[\frac{\left|B_{y}\right|}{\left|B_{z}\right|}\right] \quad \text { for } B_{z}>0
$$

In addition, we have computed the Joule energy deposited at high latitudes. It is well known that electrojet current flows in the auroral ionospheric $E$-region, and the auroral electrojet index $A E$ is used to monitor this high latitude current system. During magnetically disturbed periods, enhanced conductivities in the $E$-region produce large currents, which lead to enhanced Joule heating $\left(I^{2} R\right)$. This enhanced heating modifies the neutral wind patterns in the high latitudes, which in turn sets up the disturbance dynamo (Blanc \& Richmond, 1980). The effects of disturbance dynamo are globally observed in the Earth's atmosphere-ionosphere system (Fejer et al., 1991; Kakad et al., 2016; Kakad et al., 2017b). Even during quiet time the charged particles keep entering the high latitude polar region owing to open field configuration of the Earth's magnetic field lines. Thus the variation in auroral electrojet is observed on both short and long timescales. Akasofu (1981) proposed the following empirical formula to estimate the Joule energy using $A E$ index,

$$
E_{\text {Joule }}=2 \times A E(n T) \times 10^{8}(\mathrm{~W}) .
$$

The factor of 2 is multiplied to obtain contributions from both the hemispheres. This empirical relationship has been employed in many studies to obtain quantitative estimates of Joule heating energy (Ahn et al., 1983; Baumjohann \& Kamide, 1984; Vichare et al., 2005). For a better understanding of the low latitude magnetospheric current system, called a ring current, we have looked into variation of disturbance storm time index Dst. It is a measure of the symmetric ring current in the Earth's magnetosphere. Ring current is a westward current flowing around the Earth typically at 3-7 $R_{\mathrm{E}}$ during magnetically quiet time, and it comes closer to the Earth during the magnetically disturbed time. Injection of protons of solar wind origin from the Earth's magnetotail contributes mainly to the ring current. Although ring current enhances during periods of geomagnetic storm, it also exists during the magnetically quiet time with less magnitude.

Another current system is the EEJ, which is an eastward current flowing in a narrow latitudinal belt $\left( \pm 3^{\circ}\right)$ over the magnetic equator in the $E$-region. The hourly values of horizontal component of the ground magnetic field $(H)$ recorded at Alibag and Tirunelveli/Trivandrum are used to estimate the strength of $E E J$ on $n$th day at local time $t=1-24$ as follows (Yadav et al., 2017):

$$
\begin{aligned}
& \Delta H_{\mathrm{A}}^{n}(t)=H_{\mathrm{A}}^{n}(t)-H_{\mathrm{A}}^{n}(t=0), \\
& \Delta H_{\mathrm{T}}^{n}(t)=H_{\mathrm{T}}^{n}(t)-H_{\mathrm{T}}^{n}(t=0), \\
& \operatorname{EEJ}^{n}(t)=\Delta H_{\mathrm{T}}^{n}(t)-\Delta H_{\mathrm{A}}^{n}(t),
\end{aligned}
$$

here, $H_{\mathrm{T}}$ and $H_{\mathrm{A}}$ respectively represent the horizontal component recorded at equatorial station Tirunelveli/Trivandrum and low-latitude station Alibag. $H_{\mathrm{T}}(0)$ and $H_{\mathrm{A}}(0)$ are the values at local midnight in the corresponding longitudinal sector.

After computing all above mentioned physical parameters and energies, we estimated the monthly averaged values for corresponding parameters. Such monthly averaging removes the periodicities associated with solar rotation and the shorter timescales. Later, by applying the standard 13-month smoothing function the monthly smooth values of all these parameters are estimated to use in further analysis. It is pertinent to note that use of average values offsets the effects of day-to-day variability of Sun's activity in the proxies used in the study. As our interest is to investigate the overall energy variations at Sun, and its role in configuring the near Earth environment during the entire phase of $\mathrm{SC}$, it is reasonable to use the averaged parameters. The periods of the geomagnetic storm are important and the solar wind-magnetosphere coupling during such magnetically active periods is expected to be different. In the present paper, we cannot discuss the short timescale (minutes to days) coupling of solar wind-magnetosphere, which are particularly linked with the geomagnetic storm and substorms. This is because during the process of monthly averaging of interplanetary solar wind plasma parameters the short timescale variations get smoothed out.

\section{Results and discussion}

\subsection{Variation of solar magnetic energy}

Figure 1 shows (a) variation of monthly $\mathrm{SSN}\left(S_{\mathrm{ms}}\right)$, (b) daily variation of the solar mean magnetic field and (c) corresponding monthly smooth SMED for SCs 21-24. The vertical dotted lines represent start time (black), and peak time (red) of corresponding SCs. For SCs 21-24 the variation of monthly SSN and SMED are in good correlation with a lag of seven months and their correlation coefficient is 0.81 . Monthly smooth SMED values clearly show a substantial decrease in the magnetic energy from SC 22 to SC 24. We also observed a distinct double peak structure in SMED for the last three SCs, including the present one. This feature is consistent with noticeable asymmetry in the two peaks such that the later peak is larger compared to the earlier. The two-peak structure is also seen in the monthly smooth SSN, but the difference between them is only marginal. The presence of such dual-peak at solar maximum was seen by Gnevyshev (1967) and the interval between these two peaks is called as Gnevyshev-gap (Gnevyshev, 1977; Feminella \& Storini, 1997; Norton \& Gallagher, 2010). The time of 1 st peak $\left(t_{\mathrm{p} 1}\right)$ and 2 nd peak $\left(t_{\mathrm{p} 2}\right)$ in SMED and associated energies are given in Table 2. These two peak structures are attributed to the two surges of toroidal solar magnetic field (Georgieva, 2011). In order to quantify magnetic energies associated with each $\mathrm{SC}$, we integrated SMED from start time $t_{\mathrm{s}}$ to end time $t_{\mathrm{e}}$ for a given SC. This integrated SMED represents the momentum per unit area for a SC derived from magnetic energy. The SMED associated with both peaks, and the integrated SMED for SCs 21-24 are shown in Figure 2. A significant decrease in the integrated SMED for the past two SCs, including the recent SC 24 can be clearly noticed. The current SC 24 is yet to cease, and hence, we integrated SMED till January 2018. This integrated SMED for the SC 24 is found to be meager $49 \%$ of that of SC 
(a)
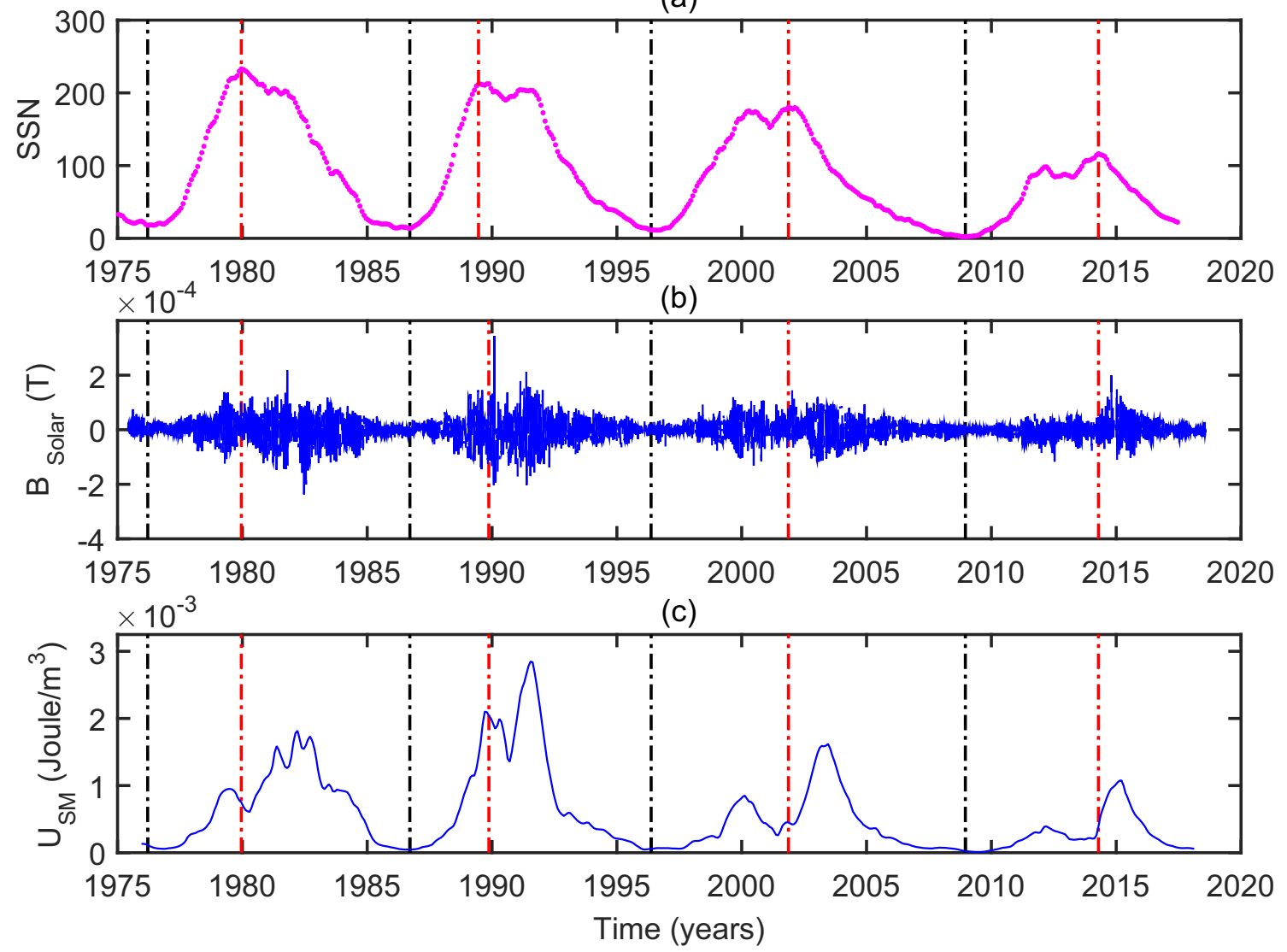

Fig. 1. Variation of (a) monthly smooth sunspot number, (b) solar magnetic field $B_{\text {solar, }}$ (c) SMED $\left(U_{\mathrm{SM}}=B_{\text {solar }}^{2} / 2 \mu_{0}\right)$ for SCs $21-24$. The vertical dashed-dotted lines indicate the time of start (black), and peak (red) for SCs.

Table 2. Occurrence time of first, and second peak in the SMED $\left(U_{\mathrm{SM}}\right)$ and corresponding values of energies are mentioned for SCs $21-24$. The SMED gives information about the average magnetic pressure. The integrated $U_{\mathrm{SM}}$ for each SC is given in last column. It may be noted that present SC 24 is not yet ended. The value $\Sigma_{t_{\mathrm{s}}}^{t_{\mathrm{e}}} U_{\text {SM }}$ for SC 24 represents the energies contribution till January 2018.

\begin{tabular}{lccccc}
\hline SC No. & Time of 1st peak, $t_{\mathrm{p} 1}(\mathrm{yr})$ & $U_{\mathrm{SM}}^{t_{\mathrm{p} 1}}\left(\mathrm{~J} / \mathrm{m}^{3}\right)$ & Time of 2nd peak, $t_{\mathrm{p} 2}(\mathrm{yr})$ & $U_{\mathrm{SM}}^{t_{\mathrm{p} 2}}\left(\mathrm{~J} / \mathrm{m}^{3}\right)$ & $\Sigma_{t_{\mathrm{s}}}^{t_{\mathrm{e}}} U_{\mathrm{SM}}\left(\mathrm{J} \mathrm{s} / \mathrm{m}^{3}\right)$ \\
\hline 21 & 1979.46 & $9.50 \mathrm{E}-04$ & 1982.21 & $1.81 \mathrm{E}-03$ \\
22 & 1989.71 & $2.10 \mathrm{E}-03$ & 1991.54 & $2.85 \mathrm{E}-03$ & $2.29 \mathrm{E} 5$ \\
23 & 2000.13 & $8.50 \mathrm{E}-04$ & 2003.46 & $1.62 \mathrm{E}-03$ \\
24 & 2012.21 & $3.91 \mathrm{E}-04$ & 2015.21 & $1.07 \mathrm{E}-03$ & $8.70 \mathrm{E} 5$ \\
\hline
\end{tabular}

23. An important point to note here is, from SC 22 onwards there is a substantial weakening of the Sun's magnetic field. For SC 22 to SC 23 the decrease in integrated magnetic field energy density was around 37\%, whereas SC 23 to SC 24 (till January 2018) it has reduced by $51 \%$. Therefore, it is reasonable to infer that Sun's magnetic field is perhaps passing through a weakening phase. In view of the above and were the predictions of weak solar activity during SC 25 to come true then the quantified energy densities cited in Table 2 would turn out to be crucial information to understand the state of solar activity. If we correlate the integrated SMED (see Table 2) and peak SSN (see Table 1) for SCs 21-24, we get a correlation coefficient of 0.93 . Although, this correlation coefficient is based on information of only four SCs, it is within the $95 \%$ statistical significance level. If forecasting about the peak SSN of upcoming SC 25 is available then based on this trend one can get some rough estimate about the integrated SMED linked with the SC 25. As the sunspots occur in the regions of strong magnetic field, a high correlation between the SSN and SMED is expected.

\subsection{Energy variations at the Earth's bow-shock}

Now, we have examined the physical conditions near the Earth's bow-shock nose. There are various satellites that record the solar wind parameter in the vicinity of the Earth's magnetopause. These observations are compiled, and provided to the scientific community by the OMNIWEB. In the present study, we have utilized these solar wind parameters of one-hour time resolution. Figure 3 shows the time variation of the interplanetary magnetic field, solar wind density, velocity, temperature at the bow-shock nose of the Earth's magnetosphere during 

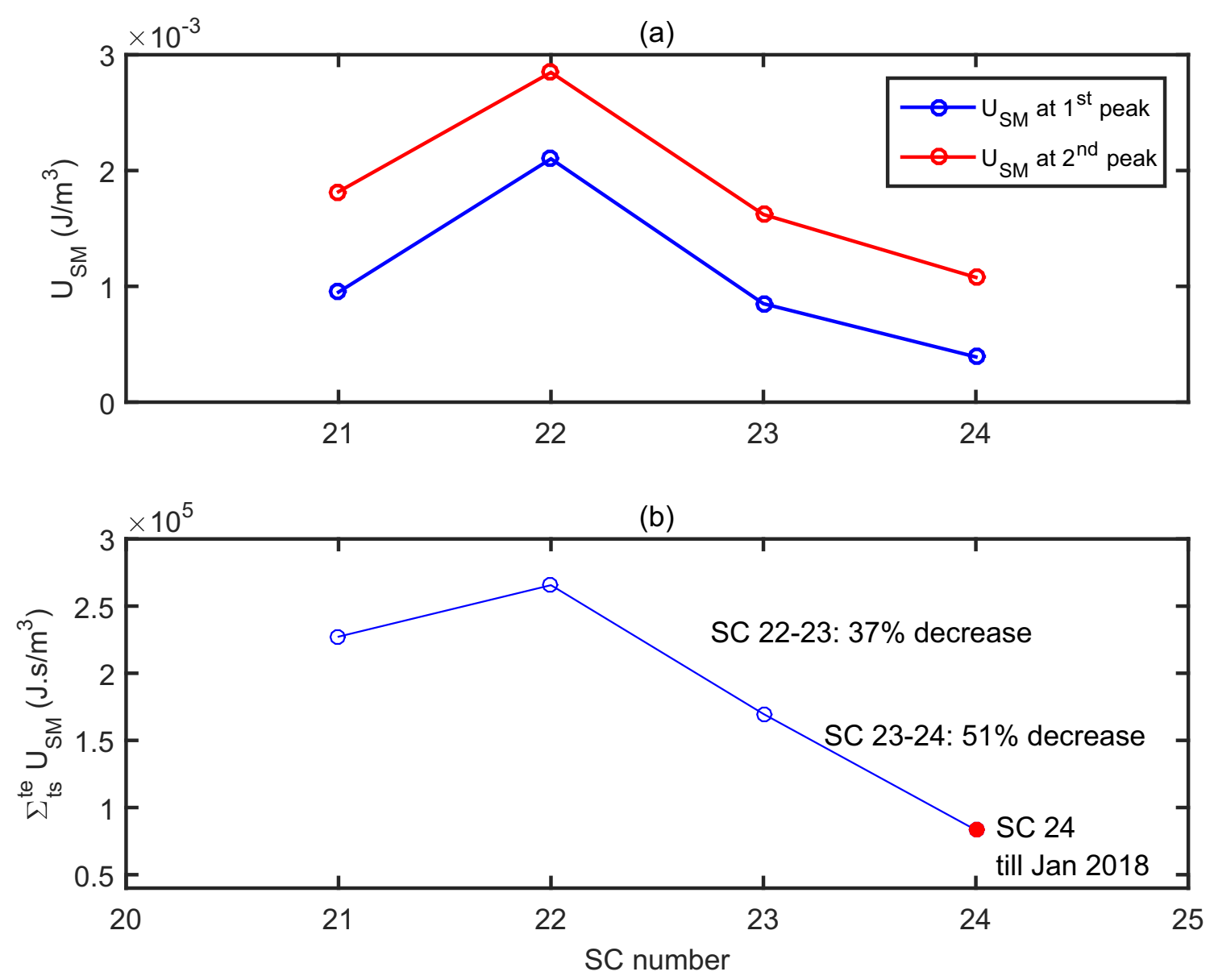

Fig. 2. (a) Variation of magnitude of 1st and 2nd peak in the SMED for SCs 21-24, (b) the integrated solar magnetic energy for SCs 21-24. The value $\Sigma_{t_{\mathrm{s}}}^{t_{\mathrm{e}}} U_{\mathrm{SM}}$ for SC 24 (shown by red solid circle) represents the integrated energy density till January 2018.

January 1985-July 2018. The range of variability in these parameters due to Sun's day-to-day activity can be seen from Figure 3. However, we explore the average behaviour of the Sun-Earth system rather than the individual events or day-today behaviour of the parameters. Thus, the 13-month smooth averages of magnetic energy density $U_{\mathrm{m}}$, kinetic energy density $U_{\text {ke, }}$, thermal energy density $U_{\text {th }}$ associated with the solar wind are plotted as a function of time in Figure 4. The corresponding standard deviations are shown by gray profiles in respective subplots. The kinetic energy density has the largest magnitude. It should be noted that variations linked to SC 22, 23 and 24 are clearly reflected in magnetic energy density as compared to thermal and kinetic energy densities. Although kinetic energy density and thermal energy density do not clearly track the monthly SSN but a gradual decrease in kinetic and thermal energy densities from SCs 22 to 24 is clearly noticed.

In-addition, to these energies, we computed monthly smooth SWP beta $(\beta)$ and magnetosonic Mach number $(M)$ at the Earth's bow-shock nose. In order to understand the role of solar activity on these parameters, we estimated the correlation coefficient between Version-2 SSN and each of these parameters. The list of parameters, and the estimated correlation coefficients are given in Table 3. We found that the SWP magnetosonic Mach number, and plasma beta are highly correlated with the monthly SSN and respectively gives a correlation coefficients of -0.92 and -0.79 . The correlation coefficient for solar wind magnetic energy density at bow-shock is 0.82 . Whereas, for other ambient SWP parameters like solar wind density, temperature, velocity, thermal energy density, and kinetic energy density, they are poorly correlated with the monthly SSN. When solar wind expands radially into the interplanetary space its density, temperature, and velocity vary during the process of expansion. Moreover, the solar wind speed changes due to acceleration/deceleration processes caused due to shocks, waveparticle interactions, etc. during its propagation. The important point is as the Sun progresses through its activity, different wind sources prevail and contribute to solar wind plasma that fills the heliosphere (Poletto, 2013). During solar maximum and outside maximum (descending/ascending phase), the likely sources for the fast solar wind are CMEs and high speed stream, respectively (Richardson et al., 2000). The variations in the source location of solar wind, interaction of fast and slow solar winds produces changes in the solar wind density, temperature and velocity. These interplanetary processes result in a lower correlation between solar wind density, velocity, and temperature with the solar activity. The low correlation between solar wind speed and sunspot number is reported by Visakh Kumar et al. (2017) for solar cycle 24.

Next, we compared the time variation of the well correlated SWP beta and magnetosonic Mach number with the monthly 

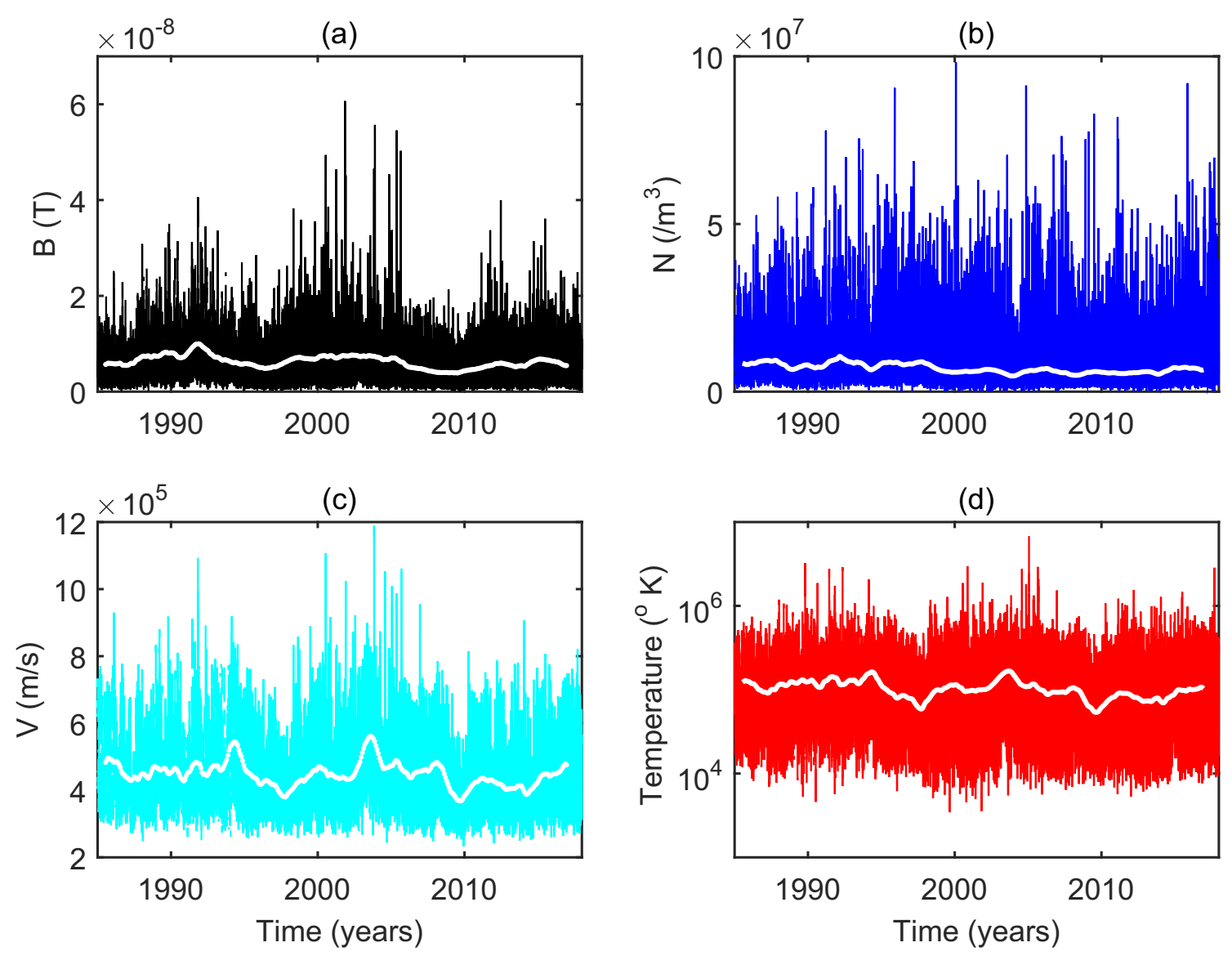

Fig. 3. Variation of (a) interplanetary magnetic field $B$, (b) SWP density $N$, (c) SWP velocity $V$, (d) proton temperature $T_{\mathrm{p}}$ of one hour resolution at bow-shock nose for SC 22, 23, and SC 24. The white solid line plotted in each panel indicate the 13-month smooth average of corresponding parameters.

SSN in Figure 5a. Here monthly SSN is plotted on a log-scale, parameter $\beta$ is multiplied by 3.5 , and magnetosonic Mach number is multiplied by a factor of 0.55 in order to have the same scales for these parameters. It is clearly evident that $\beta$ and $M$ closely follow the same time variation. It is noted that the peak in SWP beta occurs 6 months, and 15 months respectively before the solar minimum of SC 23, and 24. However, for SC 22 such time delay in the occurrence of peak SWP beta and the solar minimum is not observed. It may be noted that these time delays are obtained from the 13-month smoothed variations of corresponding quantities. One requires information for following and preceding six months ( \pm 6 months) to compute the value of smooth parameter for a given month. In that case, if the minimum in SWP beta occur much earlier (i.e., more than 6 months) then only it can be used to track the solar minimum.

It may be noted that $\beta$ and $M$ are derived from the solar wind magnetic energy density, which shows dependence on SSN. So, in Figure 5b SWP beta and in Figure 5c SWP magnetosonic Mach number is plotted as a function of monthly SSN. These variations fit well with the logarithmic curve, which are shown by the black lines in Figure $5 \mathrm{~b}$ and c. Both SWP beta and SWP magnetosonic Mach number are found to decrease as monthly SSN increases. These tendencies are governed by the following equations,

$$
\begin{gathered}
\beta=-0.25 \log \left[1+S_{\mathrm{sm}}\right]+2.13, \\
M=-0.78 \log \left[1+S_{\mathrm{sm}}\right]+10.52 .
\end{gathered}
$$

These derived relationships are based on SC 22, 23, and 24 only. It would be therefore interesting to see how SWP beta is related to future $\mathrm{SCs}$. Although maximum in $\beta$ is encountered only a few months before the solar minimum, its variation can be followed throughout the SC to get some clue of the occurrence of solar minimum. Other significance of these parameters is related to the solar wind-magnetosphere energy coupling. Earlier studies have suggested that the SWP with lower Mach numbers leads to the formation of low thermal SWP beta in the magnetosheath, which influences the magnetic forces and currents, and in turn alters the energy transfer from magnetosheath to magnetosphere. (Lavraud \& Borovsky, 2008; Pulkkinen et al., 2016). Solar wind-magnetosphere coupling efficiency is more during the periods of low Mach number 

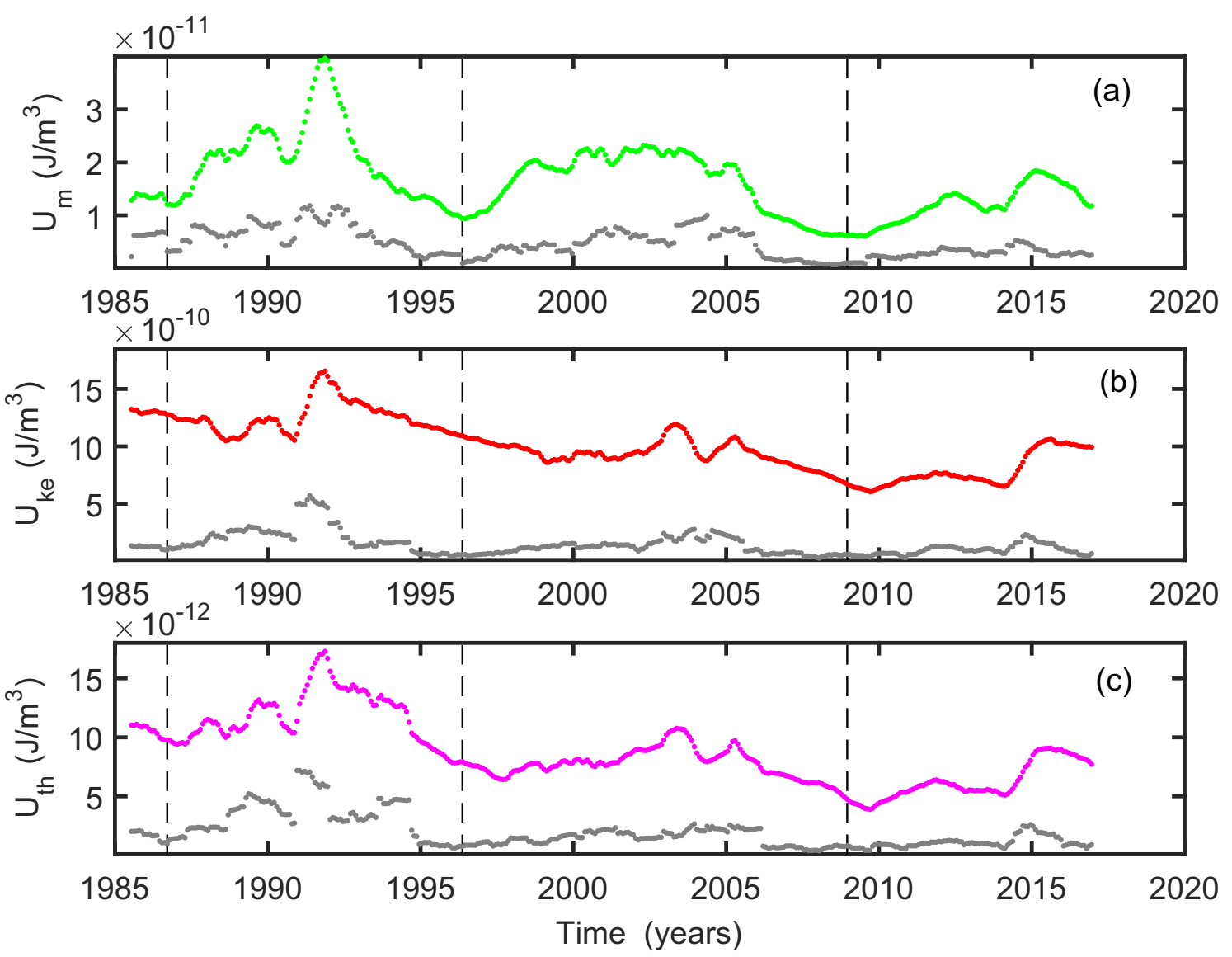

Fig. 4. The time variation of monthly smooth solar wind (a) magnetic energy density $\left(U_{\mathrm{m}}=B^{2} / 2 \mu_{0}\right)$, (b) kinetic energy density $\left(U_{\mathrm{ke}}=N m_{\mathrm{p}} V^{2} / 2\right)$, (c) thermal energy density $\left(U_{\mathrm{th}}=N K_{\mathrm{B}}\left(T_{\mathrm{p}}+T_{\mathrm{e}}\right) / 2\right)$ at Earth's bow-shock nose. The errors in the monthly smooth values of corresponding parameters are shown by gray color. Vertical black dashed lines indicate the start of SC 22, 23 and 24.

Table 3. Correlation coefficients between monthly SSN and monthly smooth magnetosonic Mach number, SWP beta, Alfven speed, energy densities at Earth's bow-shock (magnetic, thermal, and kinetic), solar wind parameters (temperature, density, and velocity), $\varepsilon, L_{\mathrm{CF}}$ and strength of different current systems (EEJ, $|D s t|, A E$, ) are given below. We used data for SCs 22, 23, and 24 to get these correlations.

\begin{tabular}{lc}
\hline Parameter & Corr. Coeff. \\
\hline$M$ & -0.92 \\
$\beta$ & -0.79 \\
$V_{\mathrm{A}}(\mathrm{m} / \mathrm{s})$ & 0.87 \\
$U_{\mathrm{m}}\left(\mathrm{J} / \mathrm{m}^{3}\right)$ & \\
$U_{\mathrm{th}}\left(\mathrm{J} / \mathrm{m}^{3}\right)$ & 0.82 \\
$U_{\mathrm{ke}}\left(\mathrm{J} / \mathrm{m}^{3}\right)$ & 0.47 \\
$T_{\mathrm{p}}\left({ }^{\circ} \mathrm{K}\right)$ & 0.27 \\
$N\left(/ \mathrm{m}^{3}\right)$ & \\
$V^{2}\left(\mathrm{~m}^{2} / \mathrm{s}^{2}\right)$ & 0.39 \\
& 0.02 \\
$\varepsilon(\mathrm{W})$ & 0.10 \\
$L_{\mathrm{CF}}\left(R_{\mathrm{E}} \mathrm{km}\right)$ & \\
$E E J(\mathrm{nT})$ & 0.85 \\
$|D s t|(\mathrm{nT})$ & -0.06 \\
$A E(\mathrm{nT})$ & 0.97 \\
\hline
\end{tabular}

(Myllys et al., 2016). The higher inverse correlation between SWP magnetosonic Mach number and monthly SSN indicate that higher solar activity can enhance the energy coupling between solar wind and magnetosphere. We have discussed the solar wind-magnetosphere energy coupling in next Section 3.3 .

From this section, we understand that the solar wind magnetic energy density $\left(U_{\mathrm{m}}\right)$, SWP beta $(\beta)$ and magnetosonic Mach number $(M)$ shows a clear dependence on SSN. Whereas, solar wind thermal $\left(U_{\text {th }}\right)$ and kinetic $\left(U_{\text {ke }}\right)$ energy densities are poorly dependent on SSN. It may be noted that the information of the $U_{\mathrm{m}}, U_{\mathrm{th}}$, and $U_{\mathrm{ke}}$ have been used to compute $\beta$ and $M$. It suggest that the observed strong dependence of SWP beta and magnetosonic Mach number on SSN is basically dictated by the solar wind magnetic energy density. Now, one needs to understand the possible link between solar wind magnetic energy density and SSN. The solar magnetic fields are frozen in the solar wind because of its high conductivity, and are dragged into the interplanetary space along with solar wind flow. It contributes mainly to the magnetic field at Earth's bow shock nose. In addition, the interplanetary coronal mass ejections (ICMEs) can contribute to the magnetic field at Earth's bow shock because these ICMEs carry magnetic flux tubes with them (Owens \& Crooker, 2006). Also, the occurrence rate of ICMEs follows the solar activity (Webb 
(a)
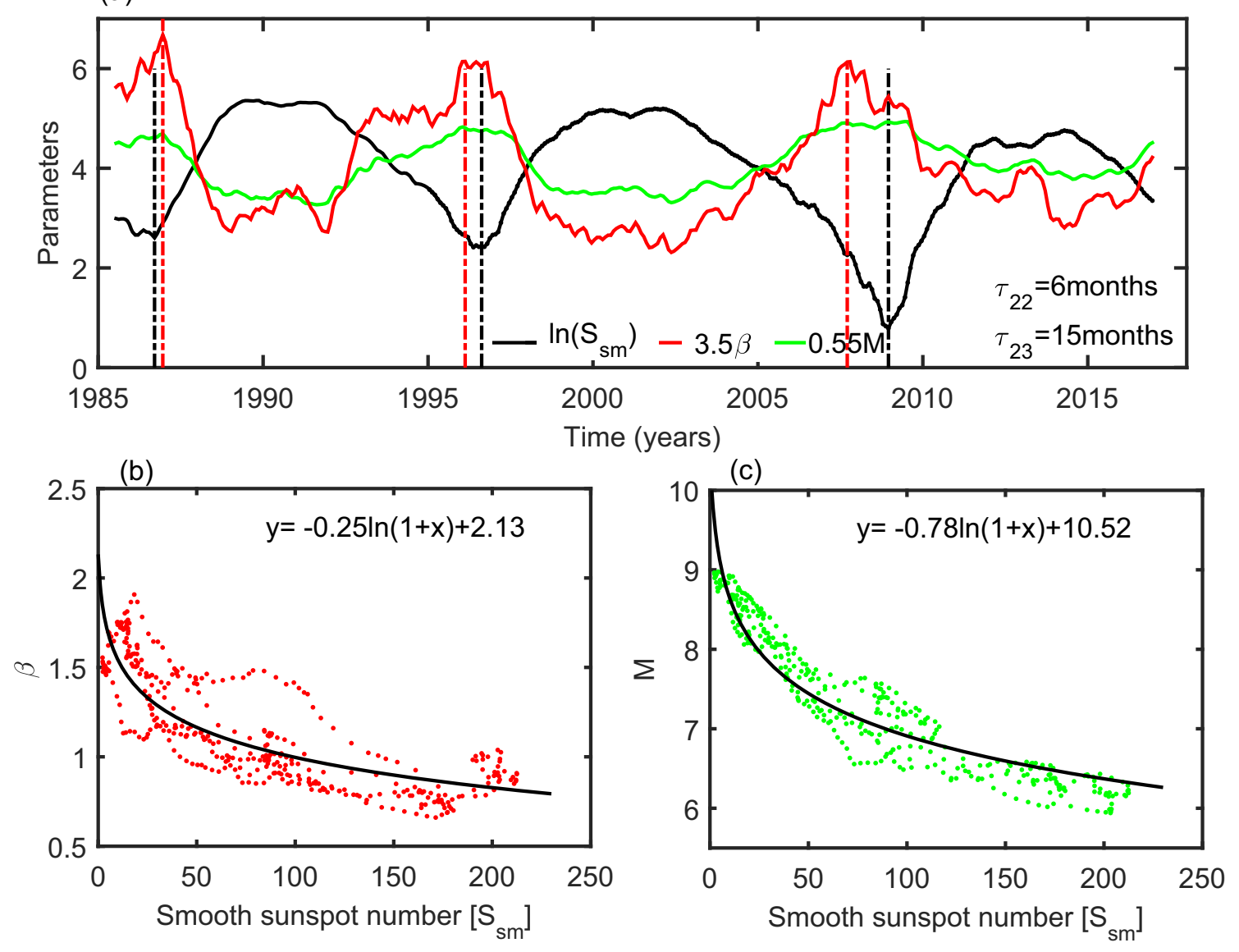

Fig. 5. (a) Comparison of monthly smooth sunspot number (black), SWP beta at the bow-shock nose (red) and solar wind magnetosonic Mach number (green). Maximum in solar plasma beta is seen few months ( $\tau=5-15$ months) before the occurrence of the solar minimum in smooth sunspot numbers. The vertical red (black) dashed lines indicate the peak in SWP beta (minimum in SSN), (b) The solar plasma beta as a function of monthly SSN for SCs 22, 23 and 24. (c) The solar wind magnetosonic Mach number as a function of monthly smooth sunspot number for SCs 22, 23, and 24. The black solid curves indicate the logarithmic fit for these variations and their equations are mentioned in respective subplots.

\& Howard, 1994). Thus, the solar wind magnetic field at bowshock is likely to follow the solar activity and hence the variations in SSN. It should be noted that variation of both closed (solar surface) and open magnetic field (interplanetary) lines has its source in the solar dynamo. So the variations in SMED and solar wind magnetic energy density at Earth's bow-shock are controlled by the solar activity.

\subsection{Solar wind-magnetosphere energy coupling}

So far, we have examined (i) what happened at the Sun and (ii) in this connection how different energies vary at the Earth's bow-shock for recent SCs. Next, we will examine the energy transferred to the Earth's magnetosphere, stand-off location of the magnetopause and various current systems in the Earth's magnetosphere-ionosphere systems. As briefed in Section 2, the Chapman-Ferraro magnetopause distance, and the solar wind-magnetosphere energy coupling parameter are estimated for SCs 22, 23, and 24 using equations (1) and (2) and their monthly smooth values are shown in Figures $6 \mathrm{a}$ and $\mathrm{b}$ respectively. The vertical dashed-dotted line indicates the start time of SC 22, 23, and 24.
The estimated position of magnetopause suggests that the magnetosphere is significantly expanded during SC 24 due to the reduced kinetic energy density (dynamic pressure). Using spacecraft observations for the SC 21, it is shown that the magnetopause stand-off distance is controlled by the solar wind dynamic pressure and their correlation coefficient is -0.70 (Petrinec et al., 1991). A recent study by Nemecek et al. (2016) also supports the dependence of magnetopause location of solar wind pressure. These observational studies support the tendency of expansion of magnetosphere during periods of reduced solar wind dynamic pressure. The magnetopause extends up to a maximum distance of $9.8 R_{\mathrm{E}}, 10.2 R_{\mathrm{E}}$, and $10.6 R_{\mathrm{E}}$, during $\mathrm{SC} 22,23$ and 24 respectively. The average stand-off location of magnetopause $\left\langle L_{\mathrm{CF}}\right\rangle$ for SC 22, 23 and SC 24 are $9.4 \pm 0.17,9.9 \pm 0.2$, and $10.2 \pm 0.3$, respectively. Equation (2) indicates that the energy coupling parameter $\varepsilon$ is proportional to $L_{C F}^{2}$. Although there is a increase in $L_{\mathrm{CF}}$ during SC 24, $\varepsilon$ encountered a substantial decrease during SC 24. The maximum power associated with $\varepsilon$ does not exceed $517 \mathrm{GW}$, $367 \mathrm{GW}$, and $281 \mathrm{GW}$ for SC 22, 23, and 24, respectively. If we integrate the solar wind energy coupling parameter $\varepsilon$ for SC 22, 23 and 24, we get $\Sigma \varepsilon=8.42 E 19 \pm 2.9 E 19(\mathrm{~J})$ for SC 
(a)

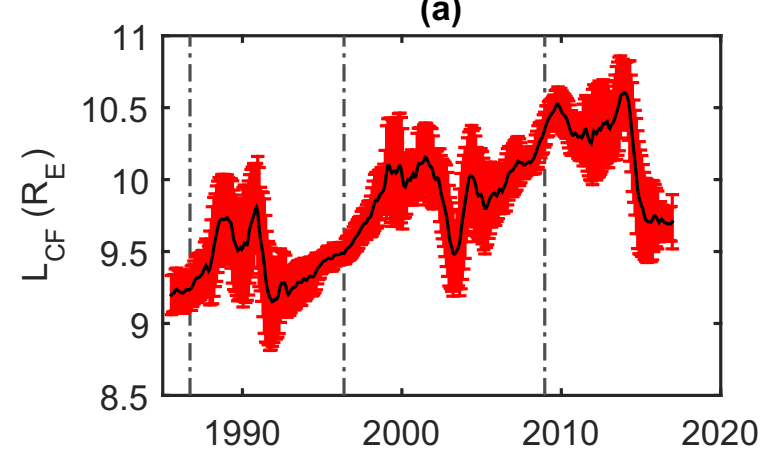

(c)

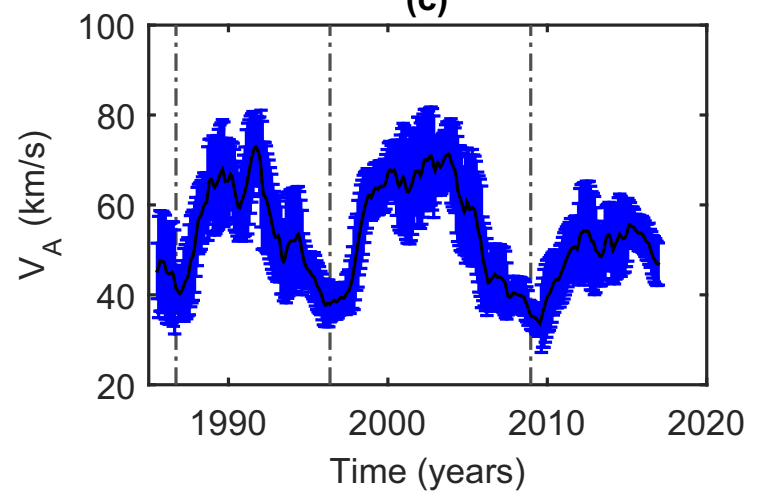

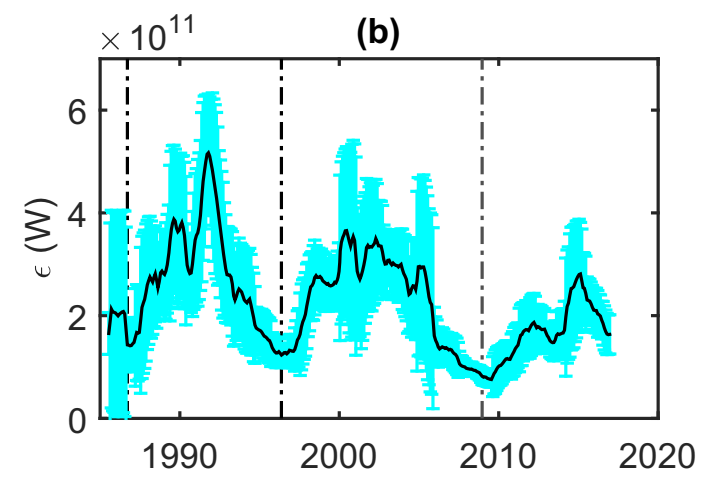

(d)

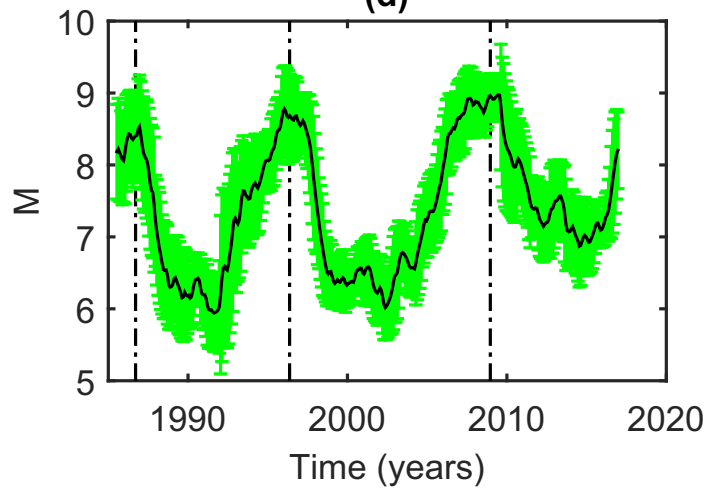

Fig. 6. Variation of monthly smooth (a) Chapman-Ferraro magnetopause distance, $L_{\mathrm{CF}}$ (b) solar wind-magnetosphere energy coupling function, $\varepsilon$, (c) Alfven speed, $V_{\mathrm{A}}$ and (d) solar wind plasma magnetosonic Mach number, $M$ for SCs 22, 23, and 24. The vertical dashed-dotted lines indicate the start of SC 22, 23, and 24. The 13-month smooth variations of these parameters are shown by black solid line.

22, and $\Sigma \varepsilon=9.2 E 19 \pm 3.1 E 19(\mathrm{~J})$ for $\mathrm{SC} 23$, and $\Sigma \varepsilon=4.23 E 19 \pm 1.3 E 19(\mathrm{~J})$ for SC 24 . The integrated solar wind-magnetosphere coupling parameter represents the total energy transferred to magnetosphere in units of Joule. So far, for the current SC 24, the energy transferred to magnetosphere is only $46 \%$ of the energy transferred during SC 23 . In Figure $6 \mathrm{c}$ and $\mathrm{d}$ the solar wind Alfven speed and magnetosonic Mach number are shown for SC 22, 23 and 24. The solar wind Alfven speed (magnetosonic Mach number) is found to be smaller (higher) during SC 24 as compared to SC 22 and SC 23. The average magnetosonic Mach number $\langle M\rangle$ for SC 22, 23 and 24 are $7.16 \pm 0.9,7.38 \pm 1$ and $7.6 \pm 0.6$, respectively. The average Alfven speed $\left\langle V_{\mathrm{A}}\right\rangle$ for SC 22, 23 and 24 are $55.1 \pm 9.9, \quad 55.5 \pm 11.9 \mathrm{~km} / \mathrm{s}$ and $48.3 \pm 6.3 \mathrm{~km} / \mathrm{s}$, respectively. Higher magnetosonic Mach number plasma is associated with higher plasma beta and it reduces the efficiency of solar wind-magnetosphere energy coupling (Lavraud \& Borovsky, 2008; Pulkkinen et al., 2016).

A noticeable weakening is seen in the solar wind magnetosphere energy coupling parameter for SC 24. In this context one needs to explore the current systems operative in the Earth's magnetosphere-ionosphere system. Here, we chose the high latitude ionospheric $E$-region electrojet current, low latitude magnetospheric ring current and equatorial ionospheric $E$-region electrojet current systems for the investigation. High latitude electrojet and ring current both are enhanced during geomagnetically disturbed periods. However at other times also, these current systems are in operation with lower magnitudes. The $A E$ index and $D s t$ index are respectively used as proxies for the high latitude electrojet, and low latitude ring currents. In addition, we have estimated the equatorial electrojet using ground magnetic field observations in the Indian longitude. Figure 7 gives the time variation of monthly smooth (a) EEJ, (b) Dst and (c) $A E$ for SCs 22, 23, and 24. The large data gap in $A E$ during 1988-1990 results in a gap in monthly smooth $A E$ plotted in Figure 7c. It is evident that the strength of ambient high latitude and equatorial electrojet, and low latitude ring current have declined during the current SC 24. The correlation coefficients between monthly SSN and monthly smoothed $A E,|D s t|$ and $E E J$ are $0.57,0.67$, and 0.97 , respectively. In earlier study it is shown that the correlation between yearly averaged solar activity and $|D s t|$ during SCs 19-23 is greater than 0.5 (Le Mouel et al., 2012). For SC 23 the minimum in Dst and maximum in $A E$ is observed in the descending phase, which could be associated with high speed streams (Tsurutani et al., 2011; Richardson \& Cane, 2012; Xystouris et al., 2014). For corresponding SCs, if we take maximum in these smoothed values as a proxy for the strength of these current systems then we find a overall reduction of $30-40 \%$ in these current systems during SC 24 as compared to $\mathrm{SC} 23$.

We have also looked into the Joule energy variation for these SCs using equation (5). We shifted the time scale to estimate the Joule power, $E_{\text {Joule }}$ such that the start time for both $\mathrm{SCs}$ coincide with each other at $\Delta t=0$. This is enabled by simply subtracting the start time $t_{\mathrm{s}}$ of corresponding SC from the time of observation $t$ (i.e., $\Delta t=t-t_{\mathrm{s}}$ ). The cumulative Joule energy is obtained integrating Joule power from $\Delta t=0$ to 
(a)

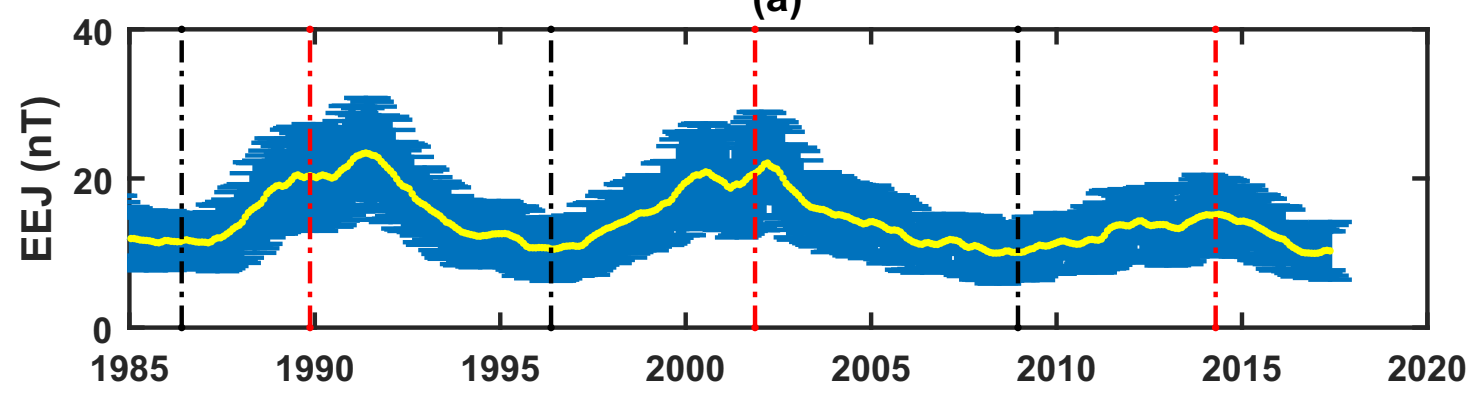

(b)

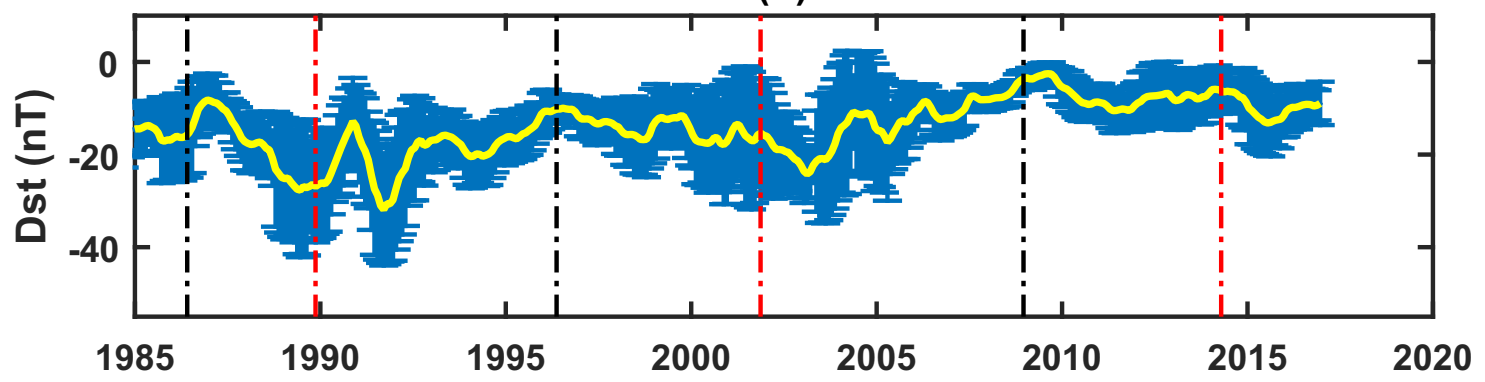

(c)

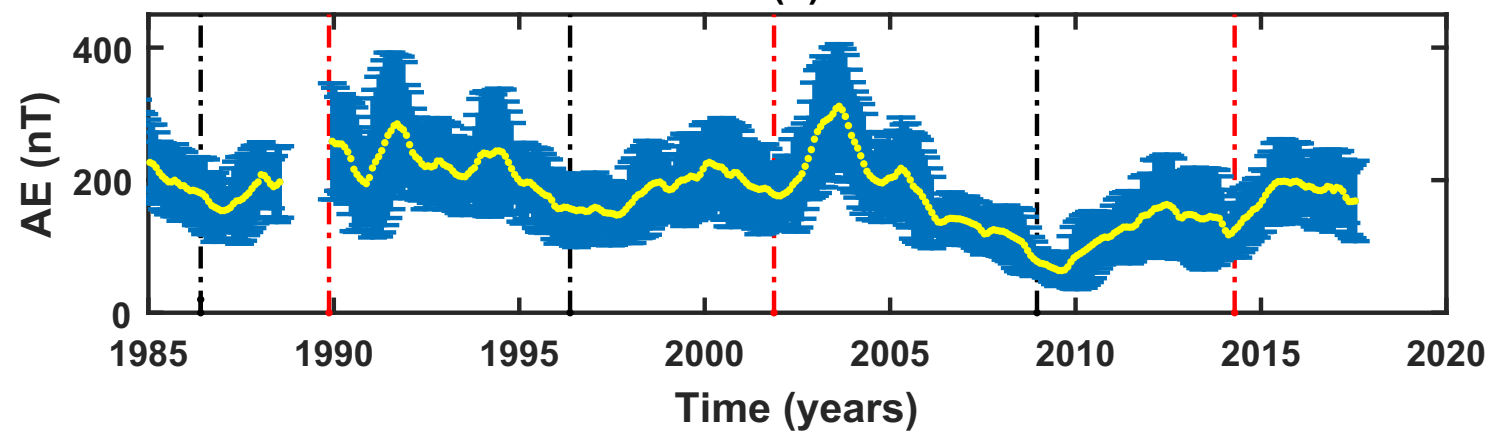

Fig. 7. Variation of monthly smooth (a) Equatorial electrojet strength in the Indian longitude (EEJ), (b) disturbed time storm current (Dst), (c) High latitude auroral electrojet current $(A E)$ as a function of time for SCs 22, 23, and 24. The 13-month smooth variations of these parameters are shown by yellow solid line. Vertical dashed-dotted lines indicate the time of start (black), and peak (red) for SCs 22, 23 , and 24.

$\Delta t=t_{\mathrm{s}}-t_{\mathrm{S}}$ for each SC. These cumulative Joule energies are plotted as a function of shifted time $\Delta t$ for SC 23, and SC 24 in Figure 8. It provides information of the deposition of Joule energy at higher latitudes as a function of time, which gives a clear evidence of significantly low Joule energy deposition at higher latitude during SC 24 as compared to SC 23 . We could not compute the integrated Joule energy for SC 22 due to large data gaps. As SC 24 has not yet ceased, we compared the values of Joule energy for $\Delta t=8$ years for both SCs. The values of total Joule energy deposited during 8 years of the initial phase of SC 23 and SC 24 are $1.03 E 19 \pm 0.32 E 19(\mathrm{~J})$, and $0.73 E 19 \pm 0.25 E 19(\mathrm{~J})$, respectively. This suggests that the Joule heating resulting from these auroral electrojet currents encountered a reduction of nearly $31 \%$ for the SC 24 . The strength of the current is decided by the ambient conductivities, and electric fields. The observed decrease in currents is attributed to the weaker ambient conductivities and electric fields during SC 24, which are driven and controlled by the Sun's energy output in the form of energetic particles and radiations. Thus, such abnormal reductions in the heating of neutral atmosphere at the high latitude domains can have several implications to our Earth's atmosphere-ionosphere-magnetosphere system.

\section{Summary and conclusions}

This study is carried out to examine the variations in the overall energies available at the Sun, bow-shock, and the Earth's upper atmosphere for recent SCs. The Sun is probably undergoing a phase of weakened activity i.e., a gradual decline in peak solar activity) during the past three SCs. Several studies have suggested that this declining phase will continue, and the upcoming SC 25 would be still weaker compared to SC 24 (Hathaway \& Wilson, 2004; Janardhan et al., 2015b). This certainly prompts us to explore whether we are heading towards solar activity akin to the Dalton or Maunder minimum. If it is affirmative, it would be interesting to be informed about the variations in the energy associated with the Sun and its influence on geospace. This would help us to understand, 


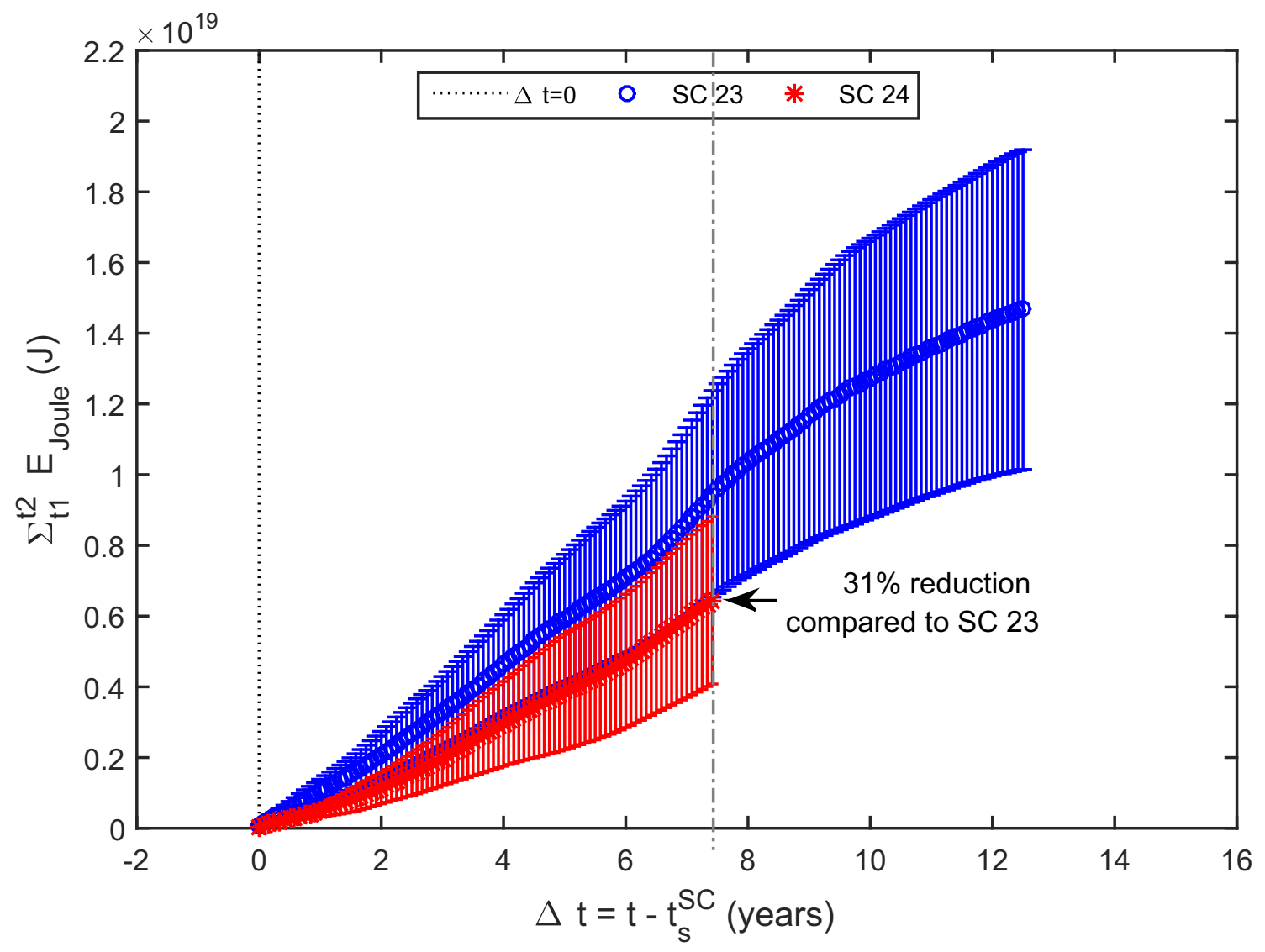

Fig. 8. Deposition of Joule energies at high latitude as SC progresses is shown for SC 23 (blue), and SC 24 (red). $\Delta t=0$ represents the start time for both SCs. 31\% reduction is seen in the high latitude Joule energy deposition for SC 24 as compared to that for SC23. The energy deposited during SC 22 is not plotted because of data gap in $A E$ during 1988-1990.

Table 4. Overall changes in different energies and physical parameters encountered during SC 24 as compared to SC 23 at Sun and near Earth environment. The quantities given in second column are mentioned with respect to the SC 23 .

\begin{tabular}{ll}
\hline Parameter & Status during SC \\
\hline$S_{\max }$ & 24 \\
$U_{\mathrm{SM}}$ & $\approx 36 \%$ reduction \\
$\Sigma_{\varepsilon}$ & $\approx 63 \%$ reduction \\
$E E J_{\max }$ & $\approx 54 \%$ reduction \\
$D s t_{\min }$ & $\approx 31 \%$ reduction \\
$A E_{\max }$ & $\approx 46 \%$ reduction \\
$\Sigma E_{\text {Joule }}$ & $\approx 36 \%$ reduction \\
$\langle M\rangle$ & $\approx 31 \%$ reduction \\
$\left\langle V_{\mathrm{A}}\right\rangle$ & $>6.9$ \\
$L_{\mathrm{CF}}$ & $<57 \mathrm{~km} / \mathrm{s}$ \\
\hline
\end{tabular}

how the Earth's ionosphere-magnetosphere systems configure themselves during periods of such low solar activity. To address this question we used various data sets available in the public domain, and estimated different energy densities at Sun, magnetosphere, and the Earth's upper atmosphere. The variation of these energies and parameters for SCs 22-24 are summarized
Table 5. Integrated energy densities and average of different parameters for SCs 22. 23, and 24 are summarized.

\begin{tabular}{lccc}
\hline Parameter & SC 22 & SC 23 & SC 24 \\
\hline$\Sigma U_{\mathrm{m}}\left(10^{-3} \mathrm{~J} \mathrm{~s} / \mathrm{m}^{3}\right)$ & $6.3 \pm 1.9$ & $6.5 \pm 1.7$ & $3.0 \pm 0.7$ \\
$\Sigma U_{\mathrm{te}}\left(10^{-3} \mathrm{~J} \mathrm{~s} / \mathrm{m}^{3}\right)$ & $3.6 \pm 1.0$ & $3.1 \pm 0.6$ & $1.6 \pm 0.3$ \\
$\Sigma U_{\mathrm{ke}}\left(\mathrm{J} \mathrm{s} / \mathrm{m}^{3}\right)$ & $0.38 \pm 0.06$ & $0.37 \pm 0.0$ & $0.20 \pm 0.03$ \\
& & & \\
$\Sigma \varepsilon\left(10^{19} \mathrm{~J}\right)$ & $8.42 \pm 2.9$ & $9.2 \pm 3.1$ & $4.23 \pm 1.3$ \\
$\Sigma E_{\mathrm{Joule}}\left(10^{19} \mathrm{~J}\right)$ & Data gap & $1.47 \pm 0.4$ & $0.72 \pm 0.26$ \\
& & & \\
$\left\langle L_{\mathrm{CF}}\right\rangle\left(R_{\mathrm{E}} \mathrm{km}\right)$ & $9.4 \pm 0.17$ & $9.9 \pm 0.2$ & $10.2 \pm 0.3$ \\
$\langle N\rangle\left(10^{6} / \mathrm{m}^{3}\right)$ & & & \\
$\left\langle T_{\mathrm{p}}\right\rangle\left(10^{5} \mathrm{~K}\right)$ & $1.18 \pm 0.2$ & $1.02 \pm 0.2$ & $0.81 \pm 0.1$ \\
$\langle V\rangle(\mathrm{km} / \mathrm{s})$ & $461 \pm 163$ & $455 \pm 187$ & $418 \pm 142$ \\
$\langle\beta\rangle$ & $1.23 \pm 0.32$ & $1.1 \pm 0.3$ & $1.07 \pm 0.18$ \\
$\langle M\rangle$ & $7.16 \pm 0.89$ & $7.38 \pm 1$ & $7.6 \pm 0.6$ \\
$\left\langle V_{\mathrm{A}}\right\rangle(\mathrm{km} / \mathrm{s})$ & $55.1 \pm 9.9$ & $55.5 \pm 11.9$ & $48.2 \pm 6.3$ \\
$\langle E E J\rangle(\mathrm{nT})$ & $16.2 \pm 4.1$ & $14.9 \pm 4$ & $12.6 \pm 1.7$ \\
$\langle D s t\rangle(\mathrm{nT})$ & $-18 \pm 5.8$ & $-13 \pm 4.1$ & $-8 \pm 2.5$ \\
$\langle A E\rangle(\mathrm{nT})$ & $211 \pm 34$ & $184 \pm 48$ & $142 \pm 39$ \\
\hline
\end{tabular}

in Table 5. Finally, this is a comprehensive study based on long term (SCs 22-24) observations. The main conclusions derived from present analysis are as follows: 
(i) Distinct double peak structures in SMED for the last four SCs (21-24) are observed. This feature is consistent with noticeable asymmetry in their two peaks. It is noted that from SC 22 to SC 23 the decrease in integrated SMED is around $37 \%$, whereas from SC 23 to SC 24 (till January 2018) it is reduced by 51\%. It indicates the substantial weakening of the Sun's magnetic field during SC 24. The weakening of the solar polar magnetic field is reported in earlier study as well (Wang et al., 2009) however, this study does not quantify the overall change in SMED.

(ii) The solar wind magnetosonic Mach number $(M)$ and SWP beta $(\beta)$ have a higher correlations $(-0.92$ and -0.79) with the SSN, compared to any other SWP parameter. This is because SWP beta and magnetosonic Mach number are derived from the interplanetary magnetic field, which seems to track the sunspot number. For SCs 23 and 24, the minimum in SWP beta $(\beta)$ is found to occur slightly earlier (6-15 months) compared to a minimum in the SSN.

(iii) The estimates of stand-off position of magnetopause for last three SCs (i.e., 22-24) suggest that the magnetosphere is likely to be significantly expanded $\left(L_{\mathrm{CF}} \leq\right.$ $\left.10.6 R_{\mathrm{E}}\right)$ during $\mathrm{SC} 24$ as compared to $\mathrm{SC} 23\left(L_{\mathrm{CF}} \leq\right.$ $\left.10.2 R_{\mathrm{E}}\right)$ and SC $22\left(L_{\mathrm{CF}}=\leq 9.8 R_{\mathrm{E}}\right)$. It is in agreement with earlier observational study that reports the expansion during periods of reduced solar wind dynamic pressure (Petrinec et al., 1991).

(iv) It is clearly evident that energy transferred to magnetosphere (i.e., $\Sigma_{\varepsilon}$ ) during the present $\mathrm{SC}$ is approximately $46 \%$ as compared to the the energy transferred during SC 23. These energy transfer estimates are based on the solar wind-magnetosphere coupling function proposed by Perreault \& Akasofu (1978), and if we use different empirical energy transfer coupling function then some deviations are expected in the quantified energy transfer to the magnetosphere during SC 24.

(v) A substantial (15-38\%) decrease in average strength of equatorial electrojet (EEJ), auroral electrojet current $A E$, and low latitude ring current (Dst) is noticed for SC 24.

(vi) The Joule heating arising from the auroral electrojet currents encountered a reduction of nearly $31 \%$ for current SC 24.

(vii) A clear difference in the solar wind Alfven speed and magnetosonic Mach number are observed for SC 23 $\left(\left\langle V_{\mathrm{A}}\right\rangle<71 \mathrm{~km} / \mathrm{s}\right)$ and SC $24\left(\left\langle V_{\mathrm{A}}\right\rangle<57 \mathrm{~km} / \mathrm{s}\right)$. The SWP magnetosonic Mach number and beta are in good agreement with a correlation coefficient of 0.96 . The low magnetosonic Mach number solar wind leads to the formation of a low thermal beta, which affects the magnetic forces and currents, and in turn modifies the solar wind-magnetosphere energy coupling.

Broadly, the present study indicates a significant step down in various energies at the Sun, magnetopause, and the Earth's atmosphere during the present SC 24, which are quantified and summarized in Table 4. This has varied implications to the Earth's atmosphere-ionosphere-magnetosphere system. The decrease in overall energies linked to magnetosphere, and the Earth's current systems are attributed to weakening in the solar output in the form of energetic particles and radiations. Finally, it would indeed be very interesting to see how SC 24 progresses during its declining phase, and record the response of geospace to the upcoming SC 25.

Acknowledgements. We thank SIDC, SILSO team for the daily international sunspot data (http://www.sidc.be/silso/), Wilcox solar observatory for solar magnetic field data (http://wso.stanford.edu/), WDC Kyoto, Japan for geomagnetic activity indices (http://wdc.kugi.kyoto-u.ac.jp/). Also, we thank OMNIWEB, NASA Space Physics Data Facility at the Goddard Space Flight Center for interplanetary parameters (https://omniweb.gsfc.nasa.gov/). We thank IIGWDC for ground magnetic field data (www.wdciig.res.in). GSL thanks the Indian National Science Academy for the support under the INSA - Honorary Scientist scheme. The revision of the paper was carried at RISH, Kyoto university Japan by BK. The editor thanks Ian Richardson and an anonymous referee for their assistance in evaluating this paper.

\section{References}

Ahn B-H, Akasofu S-I, Kamide Y. 1983. The Joule heat production rate and the particle energy injection rate as a function of the geomagnetic indices $\mathrm{AE}$ and AL. J Geophys Res Space Phys 88(A8): 6275-6287. DOI: 10.1029/JA088iA08p06275.

Akasofu S-I. 1981. Energy coupling between the solar wind and the magnetosphere. Space Sci Rev 28(2): 121-190. DOI: 10.1007/ BF00218810.

Baumjohann W, Kamide Y. 1984. Hemispherical Joule heating and the AE indices. J Geophys Res Space Phys 89(A1): 383-388. DOI: 10.1029/JA089iA01p00383.

Bisoi SK, Janardhan P, Chakrabarty D, Ananthakrishnan S, Divekar A. 2014. Changes in quasi-periodic variations of solar photospheric fields: Precursor to the deep solar minimum in cycle 23? Sol Phys 289(1): 41-61.

Blanc M, Richmond A. 1980. The ionospheric disturbance dynamo. J Geophys Res Space Phys 85(A4): 1669-1686. DOI: 10.1029/ JA085iA04p01669.

Bose S, Nagaraju K. 2018. On the variability of the solar mean magnetic field: contributions from various magnetic features on the surface of the Sun. Astrophys $J$ 862(1): 35, http://stacks. iop.org/0004-637X/862/i=1/a=35.

Clette F, Lefèvre L, Cagnotti M, Cortesi S, Bulling A. 2016. The revised Brussels-Locarno sunspot number (1981-2015). Sol Phys 291(9-10): 2733-2761.

de Toma G, Gibson S, Emery B, Arge C. 2010. The Minimum between cycle 23 and 24: Is sunspot number the whole story? In: SOHO- 23: Understanding a Peculiar Solar Minimum, vol. 428, 217, http://adsabs.harvard.edu/abs/2010ASPC.428.217D.

Dudok de Wit T, Bruinsma S. 2017. The $30 \mathrm{~cm}$ radio flux as a solar proxy for thermosphere density modelling. J Space Weather Space Clim 7: A9. DOI: 10.1051/swsc/2017008.

Ermolli I, Matthes K, Dudok de Wit T, Krivova NA, Tourpali K, et al. 2013. Recent variability of the solar spectral irradiance and its impact on climate modelling. Atmos Chem Phys 13(8): 3945 3977. DOI: 10.5194/acp-13-3945-2013, http://www.atmos-chemphys.net/13/3945/2013/.

Fejer BG, de Paula ER, Gonzlez SA, Woodman RF. 1991. Average vertical and zonal F region plasma drifts over Jicamarca. J Geophys Res Space Phys 96(A8): 13901-13906. DOI: 10.1029/91JA01171. 
Feminella F, Storini M. 1997. Large-scale dynamical phenomena during solar activity cycles. Astron Astrophys 322: 311-319.

Georgieva K. 2011. Why the sunspot cycle is double peaked. ISRN Astron Astrophys 2011.

Gkana A, Zachilas L. 2016. Re-evaluation of predictive models in light of new data: Sunspot number version 2.0. Sol Phys 291: 2457-2472. DOI: 10.1007/s11207-016-0965-3.

Gnevyshev M. 1967. On the 11-years cycle of solar activity. Sol Phys 1(1): 107-120.

Gnevyshev M. 1977. Essential features of the 11-year solar cycle. Sol Phys 51(1): 175-183.

Guo J, Feng X, Emery BA, Zhang J, Xiang C, Shen F, Song W. 2011. Energy transfer during intense geomagnetic storms driven by interplanetary coronal mass ejections and their sheath regions. $J$ Geophys Res Space Phys 116(A5).

Hajra R, Tsurutani BT, Echer E, Gonzalez WD, Gjerloev JW. 2016. Supersubstorms ( $\left.\mathrm{SML}_{\mathrm{i}} 2500 \mathrm{nT}\right)$ : Magnetic storm and solar cycle dependences. J Geophys Res Space Phys 121(8): 7805-7816, 2015JA021835. DOI: 10.1002/2015JA021835.

Hathaway DH, Upton L. 2014. The solar meridional circulation and sunspot cycle variability. J Geophys Res Space Phys 119(5): 3316-3324. DOI: 10.1002/2013JA019432.

Hathaway DH, Wilson RM. 2004. What the sunspot record tells us about space climate. Sol Phys 224(1-2): 5-19. DOI: 10.1007/ s11207-005-3996-8.

Janardhan P, Bisoi SK, Gosain S. 2010. Solar polar fields during cycles 21-23: Correlation with meridional flows. Sol Phys 267(2): 267-277.

Janardhan P, Bisoi SK, Ananthakrishnan S, Tokumaru M, Fujiki K. 2011. The prelude to the deep minimum between solar cycles 23 and 24: Interplanetary scintillation signatures in the inner heliosphere. Geophys Res Lett 38(20).

Janardhan P, Bisoi SK, Ananthakrishnan S, Tokumaru M, Fujiki K, Jose L, Sridharan R. 2015a. A 20 year decline in solar photospheric magnetic fields: Inner-heliospheric signatures and possible implications. J Geophys Res Space Phys 120(7): 53065317.

Janardhan P, Bisoi SK, Ananthakrishnan S, Tokumaru M, Fujiki K, Jose L, Sridharan R. 2015b. A 20 year decline in solar photospheric magnetic fields: Inner-heliospheric signatures and possible implications. J Geophys Res Space Phys 120(7): 53065317. DOI: $10.1002 / 2015 J A 021123$.

Kakad B. 2011. A new method for prediction of peak sunspot number and ascent time of the solar cycle. Sol Phys 270(1): 393406. DOI: $10.1007 / \mathrm{s} 11207-011-9726-5$.

Kakad B, Gurram P, Tripura Sundari PNB, Bhattacharyya A. 2016. Structuring of intermediate scale equatorial spread $\mathrm{F}$ irregularities during intense geomagnetic storm of solar cycle 24. J Geophys Res Space Phys 121(7): 7001-7012, 2016JA022635. DOI: 10.1002/2016JA022635.

Kakad B, Kakad A, Ramesh DS. 2017a. Shannon Entropy-Based Prediction of Solar Cycle 25. Sol Phys 292: 95-107. DOI: 10.1007/s11207-017-1119-y.

Kakad B, Surve G, Tiwari P, Yadav V, Bhattacharyya A. 2017b. Disturbance dynamo effects over low-latitude F region: A study by network of VHF spaced receivers. J Geophys Res Space Phys 122(5): 5670-5686, 2016JA023498. DOI: 10.1002/ 2016JA023498.

Koskinen HE, Tanskanen EI. 2002. Magnetospheric energy budget and the epsilon parameter. $J$ Geophys Res Space Phys 107(A11).

Lavraud B, Borovsky JE. 2008. Altered solar wind-magnetosphere interaction at low Mach numbers: Coronal mass ejections.
$J$ Geophys Res Space Phys 113(A9), 1-25, A00B08: DOI: 10.1029/2008JA013192.

Le G, Cai Z, Wang H, Zhu Y. 2012. Solar cycle distribution of great geomagnetic storms. Astrophys Space Sci 339(1): 151-156.

Le Mouel J-L, Blanter E, Shnirman M, Courtillot V. 2012. On secular changes of correlation between geomagnetic indices and variations in solar activity. $J$ Geophys Res Space Phy 117(A9).

Li K, Yun H, Gu X. 2001. On long-term predictions of the maximum sunspot numbers of solar cycles 21-23. Astron Astrophys 368(1): 285-291.

Manoharan P. 2012. Three-dimensional evolution of solar wind during solar cycles 22-24. Astrophys J 751(2): 128.

McComas D, Ebert R, Elliott H, Goldstein B, Gosling J, Schwadron N, Skoug R. 2008. Weaker solar wind from the polar coronal holes and the whole Sun. Geophys Res Lett 35(18). DOI: 10.1029/ 2008 GL034896.

Myllys M, Kilpua E, Lavraud B, Pulkkinen T. 2016. Solar windmagnetosphere coupling efficiency during ejecta and sheathdriven geomagnetic storms. $J$ Geophys Res Space Phys 121(5): 4378-4396.

Nandy D, Munoz-Jaramillo A, Martens PC. 2011. The unusual minimum of sunspot cycle 23 caused by meridional plasma flow variations. Nature 471(7336): 80.

Nemecek Z, Safrankova J, Lopez R, Dusik S, Nouzak L, Pech L, Simnek J, Shue J-H. 2016. Solar cycle variations of magnetopause locations. Adv Space Res 58(2): 240-248.

Newbury JA, Russell CT, Phillips JL, Gary SP. 1998. Electron temperature in the ambient solar wind: Typical properties and a lower bound at 1 AU. J Geophys Res Space Phys 103(A5): 9553 9566. DOI: 10.1029/98JA00067.

Newell P, Sotirelis T, Liou K, Meng C-I, Rich F. 2007. A nearly universal solar wind-magnetosphere coupling function inferred from 10 magnetospheric state variables. J Geophys Res Space Phys 112(A1).

Norton A, Gallagher J. 2010. Solar-cycle characteristics examined in separate hemispheres: Phase, Gnevyshev gap, and length of minimum. Sol Phys 261(1): 193.

Owens MJ, Crooker N. 2006. Coronal mass ejections and magnetic flux buildup in the heliosphere. J Geophys Res Space Phys 111(A10).

Perreault P, Akasofu S. 1978. A study of geomagnetic storms. Geophys J Int 54(3): 547-573.

Petrinec S, Song P, Russell C. 1991. Solar cycle variations in the size and shape of the magnetopause. J Geophys Res Space Phys 96(A5): 7893-7896.

Petrovay K. 2010. Solar cycle prediction. Liv Rev Sol Phys 7(1): 6.

Poletto G. 2013. Sources of solar wind over the solar activity cycle. $J$ Adv Res 4(3): 215-220.

Pulkkinen TI, Dimmock AP, Lakka A, Osmane A, Kilpua E, Myllys M, Tanskanen EI, Viljanen A. 2016. Magnetosheath control of solar wind-magnetosphere coupling efficiency. $J$ Geophys Res Space Phys 121(9): 8728-8739, 2016JA023011. DOI: 10.1002/ 2016JA023011.

Richardson IG. 2013. Geomagnetic activity during the rising phase of solar cycle 24. J Space Weather Space Clim 3: A08.

Richardson IG, Cane HV. 2012. Near-earth solar wind flows and related geomagnetic activity during more than four solar cycles (1963-2011). J Space Weather Space Clim 2: A02.

Richardson I, Cliver E, Cane H. 2000. Sources of geomagnetic activity over the solar cycle: Relative importance of coronal mass ejections, high-speed streams, and slow solar wind. J Geophys Res Space Phys 105(A8): 18203-18213. 
Schrijver CJ, Liu Y. 2008. The global solar magnetic field through a full sunspot cycle: Observations and model results. Sol Phys 252(1): 19-31.

Selvakumaran R, Veenadhari B, Akiyama S, Pandya M, Gopalswamy N, Yashiro S, Kumar S, Mkel P, Xie H. 2016. On the reduced geoeffectiveness of solar cycle 24: A moderate storm perspective. J Geophys Res Space Phys 121(9): 8188-8202, 2016JA022885. DOI: 10.1002/2016JA022885.

Sibeck DG, Lopez RE, Roelof EC. 1991. Solar wind control of the magnetopause shape, location, and motion. J Geophys Res Space Phys 96(A4): 5489-5495. DOI: 10.1029/90JA02464.

Solomon SC, Qian L, Burns AG. 2013. The anomalous ionosphere between solar cycles 23 and 24. J Geophys Res Space Phys 118(10): 6524-6535. DOI: 10.1002/jgra.50561.

Tsurutani BT, Gonzalez WD, Lakhina GS, Alex S. 2003. The extreme magnetic storm of 12 September 1859. J Geophys Res Space Phys 108(A7), 1-8, 1268: DOI: 10.1029/2002JA009504.

Tsurutani BT, Echer E, Guarnieri FL, Gonzalez WD. 2011. The properties of two solar wind high speed streams and related geomagnetic activity during the declining phase of solar cycle 23 . J Atmos Sol-Terr Phys 73(1): 164-177.

Vichare G, Alex S, Lakhina G. 2005. Some characteristics of intense geomagnetic storms and their energy budget. $J$ Geophys Res Space Phys 110(A3).
Visakh Kumar U, Varghese B, Kurian PJ. 2017. Relation between solar wind parameters, coronal mass ejections and sunspot numbers. Int J Eng Appl Sci 4(9): 2394-3661.

Wang Y-M, Robbrecht E, Sheeley N Jr. 2009. On the weakening of the polar magnetic fields during solar cycle 23. Astrophys $J$ 707(2): 1372.

Webb DF, Howard RA. 1994. The solar cycle variation of coronal mass ejections and the solar wind mass flux. $J$ Geophys Res Space Phys 99(A3): 4201-4220.

Wing S, Johnson JR, Vourlidas A. 2018. Information theoretic approach to discovering causalities in the solar cycle. Astrophys $J$ 854(2): 85.

Xystouris G, Sigala E, Mavromichalaki H. 2014. A complete catalogue of high-speed solar wind streams during solar cycle 23 . Sol Phys 289(3): 995-1012.

Yadav V, Kakad B, Bhattacharyya A, Pant TK. 2017. Quiet and disturbed time characteristics of blanketing Es (Esb) during solar cycle 23. J Geophys Res Space Phys 122(11): 11591-11606, 2017JA023911. DOI: 10.1002/2017JA023911.

Yamauchi M. 2015. Decreased Sun-Earth energy-coupling efficiency starting from 2006. Earth Planet Space 67(1): 44.

Zachilas L, Gkana A. 2015. On the verge of a grand solar minimum: A second maunder minimum? Sol Phys 290(5): 1457-1477. DOI: 10.1007/s11207-015-0684-1.

Cite this article as: Kakad B, Kakad A, Ramesh D.S \& Lakhina G.S 2019. Diminishing activity of recent solar cycles (22-24) and their impact on geospace. J. Space Weather Space Clim. 9, A1. 